\title{
Regression Analysis of ANN Training Algorithms for an Anaerobic Wastewater Treatment System
}

\author{
S.Harikishore, Dr.S.Shanthi
}

\begin{abstract}
The process of an anaerobic reactor in predicting the COD Level was modeled by the application of Neural Networks. The effluent COD is the most common factor used in all types of Wastewater Treatment Systems. This research is about the prediction of the concentration of COD by using the ANN, a simple feed forward, Back Propagation (BP) neural network. The three layered feed forward ANN has been trained using four back propagation algorithms. The efficiency and accuracy were measured based on MSE and Regression Coefficient(R) to evaluate their performance. The model trained with Levenberg Marquardt algorithm provided best results with $\mathrm{MSE}=0.533$ and $\mathrm{R}=0.991$. The model performed up to the expectation and the result of the prediction is appropriate.
\end{abstract}

Index Terms - Wastewater Treatment System (WWTS), Anaerobic Reactor, Chemical Oxygen Demand (COD), Artificial Neural Networks (ANN), Back-Propagation, Levenberg-Marquardt Algorithm, Gradient Descent with Adaptive Learning Rate Algorithm, Gradient Descent with Momentum Algorithm, Resilient Back-Propagation Algorithm.

\section{INTRODUCTION}

According to the great classic text 'Thirukkural', Water is known as the true ambrosial food of all the lives. Water has been used for various purposes consequently producing sewage from the households, commercial and economic sectors. This wastewater needs proper intervention before discharging them into the water bodies since the sewage contains numerous of harmful toxins and pathogens causing serious calamities [1]. Thus, the Wastewater Treatment System (WWTS) has been popularly implemented in physical, biological and chemical progressions. Thus, the linear mathematical models have been proven to be inappropriate for these non-linear wastewater processes [2].

The well- known process for the decaying of domestic and industrial sewage is through the microbial activity in the absence of oxygen, called the Anaerobic Digestion [3]. The Anaerobic reactors produce a combination gas of methane and carbon dioxide called the biogas, a good source of renewable energy [4]. Studies have been carried out to

Manuscript revised October 25, 2019 and published on November 10 , 2019

S.Harikishore, Student, PSG College of Technology, Coimbatore, India. Dr.S.Shanthi, Civil Department, Avinashilingam University for Women, Coimbatore, India. maximize the production of Biogas through various techniques. The methanogenic bacteria are involved to produce massive amount of biogas from assorted solid and water wastes [5]. Thus the flourish process has paid scope for the development of various anaerobic reactors.

This paper studies about the design of the prediction model to find the COD level in an anaerobic wastewater reactor. The inconsistency of the influent are recognized with the alteration of specific parameters' composition, strength and flow rates levels [6]. The Chemical Oxygen Demand (COD) has been used as the indicator to determine the cause of the effluents [7]. In WWTS, the water is consider more toxic to the ecological life and would be a great threat to the marine lives if the concentration of COD is higher [8]. The Anaerobic reactors forms granular sludge in minimum time which can thus helps better in the COD deduction from the affluent water [9].

The paper is about the implementation of the four different back-propagation ANN algorithms on the prediction of the COD value. The performances are compared by means of their regression and mean square values.

The stream-flow forecasting and the cohesion less soils, lateral stress resolve studied by Ozgur Kisi et al [10] has compared three back propagation Neural Network training algorithms namely Levenberg-Marquardt, Conjugate gradient and Resilient Back Propagation algorithms. The study has been concluded with result of convergence speed and the estimation accuracy. In which Levenberg-Marquardt has proven to be the best in terms of the convergences while Resilient Back Propagation has performed accurately at the determination.

Likewise Rama Subbanna et al [11], has also implemented the above mentioned three ANN training algorithms to observe the saturation level in the magnetic core of a welding transformer. Various performance metrics such as computational time, algorithm complexity, root square error and the gradient are used to determine the finest algorithm. Accordingly, Resilient Back Propagation algorithm has been chosen to be the best with less computational time and good algorithm complexity and also Levenberg-Marquardt algorithm has also proven to be good.

\section{MODEL DEVELOPMENT}

The Fig 2 shows the schematic diagram during the model development process. During the design of the model, an appropriate development tool, MATLAB has been employed. The ANN being a mathematical development modeling tool becomes an admirable platform for various learning and optimization algorithms. 


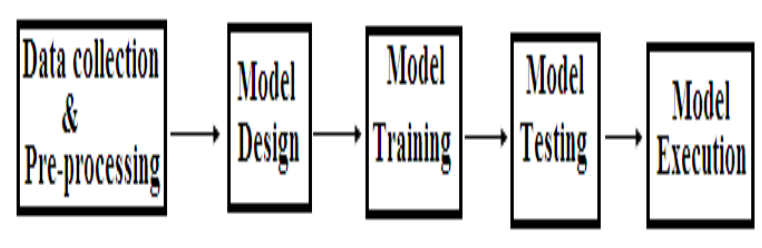

Fig 1.Systematic Flow Diagram of the Model

\section{A. Data Preprocessing}

The quality of the data has been screened for the detection of instability in the source data that might be caused because of the electromagnetic disturbance, miscalculation of the data records, uncertain errors, etc. Thus the data has been pre-processed crucially before the analytic proceedings.

\section{B. Recognizing and removing/replacing Outliers}

The mean or the median values of the data are widely used to remove/replace the missing values. When the total of WWTS parameters has minimum empty values, the Regression becomes a preeminent method. The trimmed means can be used to hold the outliners in the data. [12].Here K-Means clustering has been taken to remove the blankness and then clusters has been used to collect the interrelated data for processing [13].

\section{K-Means Clusters}

The K-Means a clustering technique with a collection of samples with specific measures of distance to form ' $\mathrm{k}$ ' number of clusters, based on a defined distance measurements [14]. K-Means frequently uses the partition algorithm with square-error criterion, which helps to minimize the following error function

$$
\begin{gathered}
\mathrm{C} \\
E=\sum \sum\left\|x_{i}-c_{k}\right\|^{2} . \\
K=1 x \varepsilon Q_{k}
\end{gathered} .
$$

where $\mathrm{C}$ is the number of clusters, ck is the centre of cluster $\mathrm{k}$ and $\mathrm{x}$ is a data sample that belongs to cluster $\mathrm{Qk}$.

The effluent COD values obtained from the anaerobic digester are used analyse by the K- Means algorithm in Java. From the initial partitions of the clusters, the centre values are calculated therefore the minimizing the square error function.

The total numbers of records accounted are about 200 and the pre-processing time has been expressed in nanoseconds. In this work, it takes 78 nanoseconds to remove/replace the missing for 61 records. With pre-processing time taken for analysis of the given data samples the K-Means has been resulted as the graphical vision.

\section{Model Design Artificial Neural Network}

The ANN is used as the prediction and forecasting modeling tool which have a equivalent ability of the human brain. There are massive amount of unified, highly processing neurons working simultaneously to resolve simple to complex problems. The Feed- Forward Back-Propagation is the supervised learning ANN algorithm is known best in the field for the calculation and updating the predicting models with minimum error [15].

\section{Model Training using BPN}

The four different Artificial Neural Network (ANN) Back Propagation training algorithms accounted in this work are Levenberg - Marquardt (LM), Gradient Descent with Adaptive Learning (GDA), Gradient Descent with Momentum (GDM) and Resilient Back-Propagation (RP) [16].

The ANN model has been trained to consider the influent COD levels and the controlling parameters as the input while consequent effluent COD levels are predicted as the output. In order to optimize the model and to have minimum error function, the model has been well trained with the flow rate value, influent COD concentration and OLR training subset. The hidden and output layers of the network are activated by using the tansig and purelin functions respectively [17].

The performance model has been evaluated by using the MSE and Regression Coefficient values.

\section{EXPERIMENTAL RESUltS}

With the learning ability the model adjusts the weights to predict the expected result; the network has been trained with four different back propagation training algorithms.

The model has been tested and the performance has been evaluated at each trial by means of the MSE and R. The performance graphs for the algorithms are shown in Fig.2, Fig.3, Fig.4 and Fig 5. The Regression (R) values for the respective algorithms are shown Fig. 6, Fig.7, Fig. 8, and Fig. 9.

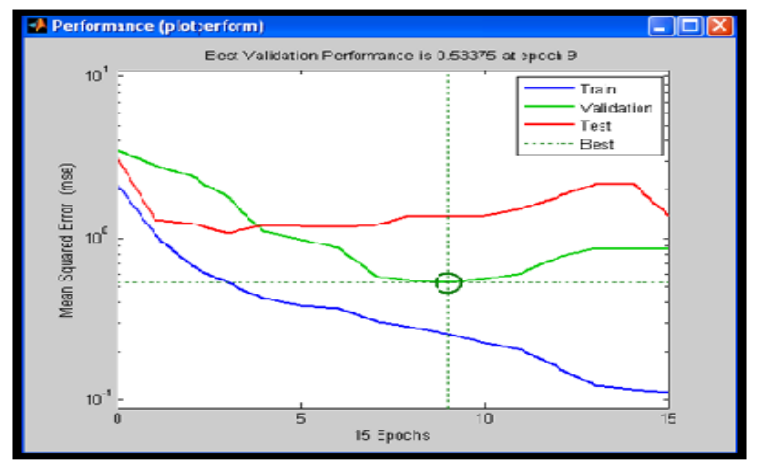

Fig 2. Performance Graph with MSE for Levenberg- Marquardt Algorithm

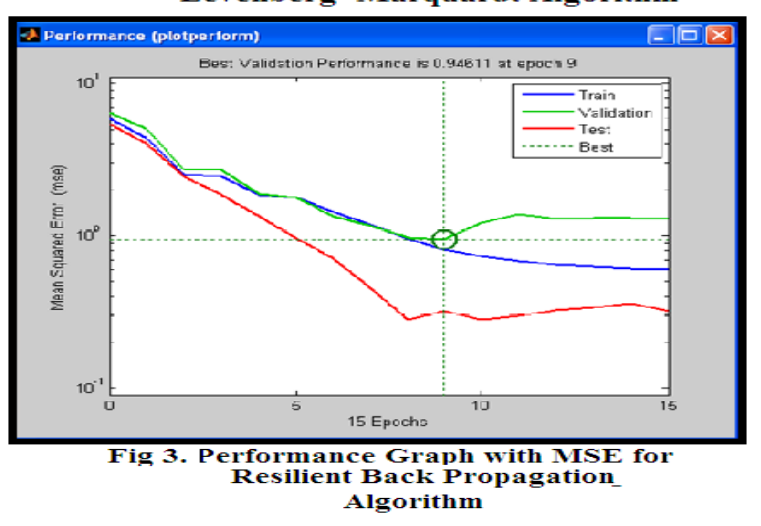




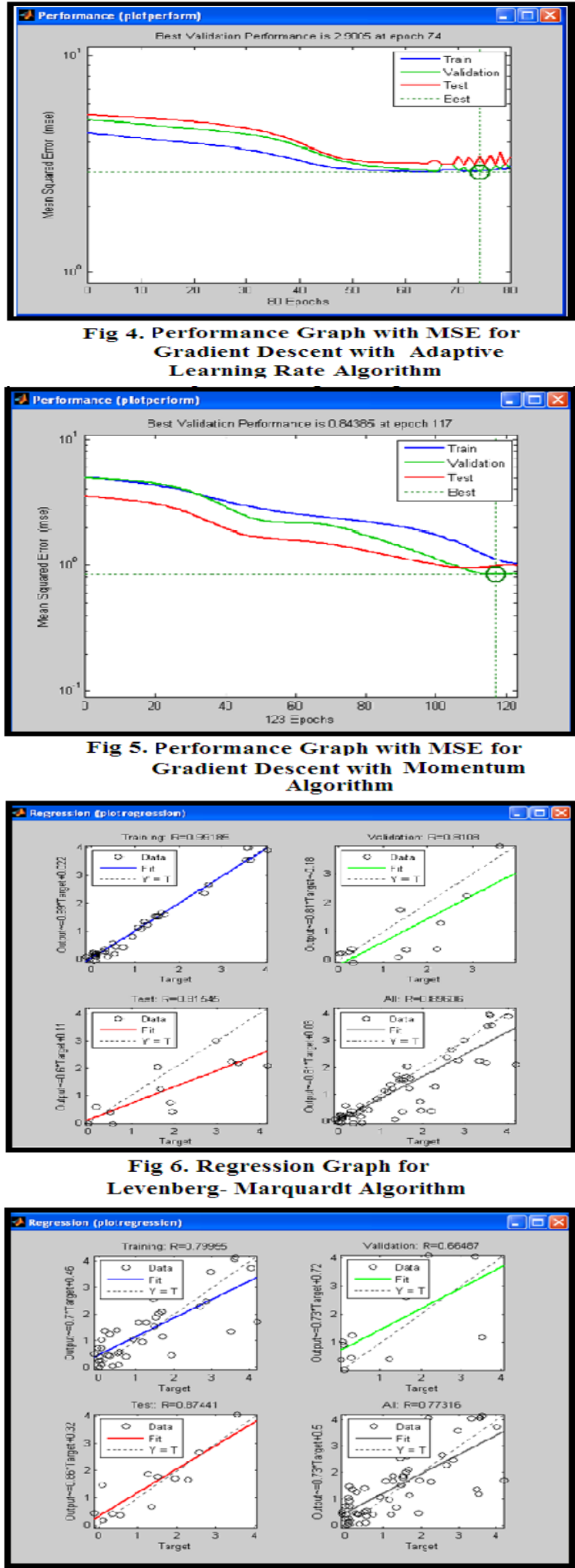

Fig 7. Regression Graph for Resilient Back Propagation Algorithm

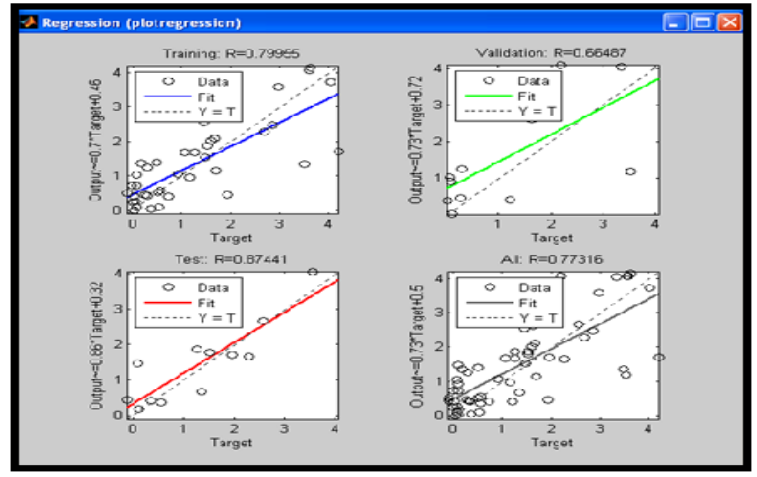

Fig 7. Regression Graph for

Resilient Back Propagation Algorithm

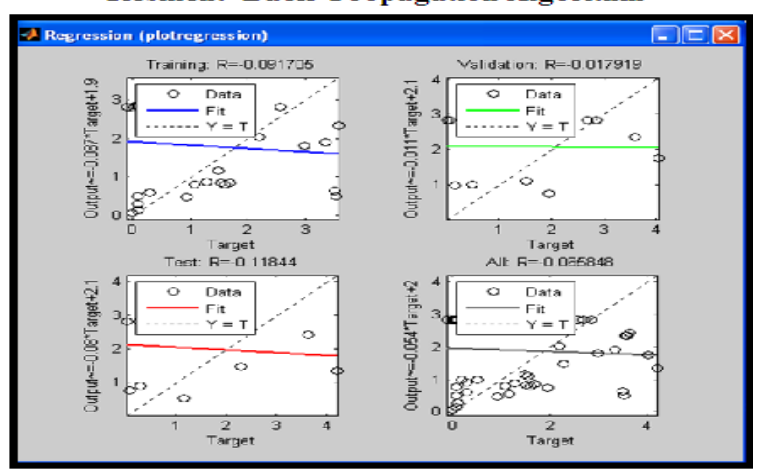

Fig 8. Regression Graph for
Gradient Descent with Adaptive Learning Rate Algorithm

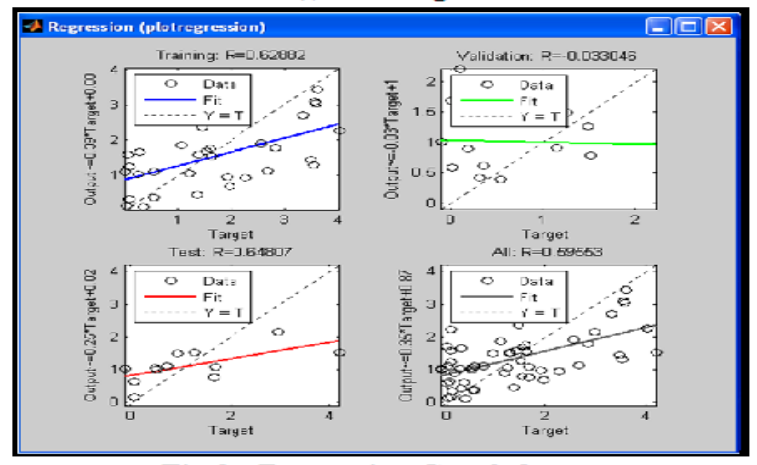

Fig 9. Regression Graph for Momentum Algorithm

\section{Performance Measures}

The four training algorithms were applied on the network model based on the inputs considered in order to predict the target COD levels. The LM algorithm has performed well by minimizing the errors and with the minimum MSE the accuracy of the prediction has been satisfactory. The $\mathrm{R}$ value acquired as the result in the prediction of resultant output has been proved to be the finest match of the network model. The Table 1 proves the performance of the network obtained for LM algorithm.

\begin{tabular}{|c|c|c|}
\hline Algorithms & Regression $(\mathrm{R})$ & MSE \\
\hline LM & 0.991 & 0.533 \\
\hline GDA & 0.092 & 2.90 \\
\hline GDM & 0.629 & 0.843 \\
\hline RP & 0.798 & 0.946 \\
\hline
\end{tabular}

Thus the network response for accurate output was calculated using the statistical indices viz., MSE and R. 


\section{Conclusion}

In this paper, the four back propagation training algorithms on the ANN model are trained and tested to forecast the level of COD. Among the four algorithms, Levenberg- Marquardt (LM) has performed well with fast convergence and the prediction has been found satisfactory. In regard to the Regression and MSE, the model when developed by using LM algorithm has showed an accurate prediction of COD level..

\section{REFERENCES}

[1] Maged M. Hamed, Mona g. Kahalafallah and Ezzat A. Hassanien, "Prediction of Wastewater Treatment Plant Performance using Artificial Neural Networks", Environmental Modeling and Software, Vol 19, Pp: 919-928, October 2004.

[2] I. Plazl, G. Pipus, M. Grolka, T.Koloini, "Parametric Sensitivity and Evaluation of a Dynamic Model for Single-Stage Wastewater Treatment Plant”, Acta Chimica Slovenica, Pp:289 - 300, Jan 1999.

[3] M.Dixon, J.R. Gallop, S. C. Lambert and J.V. Healy, "Experience with Data Mining for the Anaerobic Wastewater Treatment Process", Environmental Modeling and Software, Vol 22, Pp: 315-322, March 2007.

[4] Olivier Bernard, Zakaria Hadj-Sadok, Denis Dochain, Antoinc Genovesi, Jean-Philippe Steyer, "Dynamic Model Development and Parameter Identification for an Anaerobic Wastewater Treatment Process", Biotechnology and Bioengineering, Vol15 (4), Pp:424438, June 2001.

[5] Fasosin Emmanuel Olufemi, David Veronica and Hero Godwin , "Effect of Anaerobic Co-digestion on Microbial Community and Biogas Production", Biosciences Biotechnology Research Asia, Vol 16(20), Pp: 391-401, June 2019.

[6] Hamoda M.F, Al-Gusain .I. A., A. H. Hassan,, 'Integrated Wastewater Treatment Plant Performance Evaluation using Artificial Neural Network", Water Science and Technology, Vol 40, Pp: 55-69, 1999.

[7] Colin M.W. Harnadek, Nigel G.H. Guilford and Dr. Elizabeth A. Edwards, "Chemical Oxygen Demand Analysis of Anaerobic Digester Contents", Stem Fellowship Journal, Vol 1, Issue 2, Pp: 1-5, Dec 2015

\section{AUTHORS PROFILE}

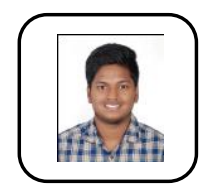

S.Harikishore, Engineering student of PSG College of Technology, Coimbatore, India.

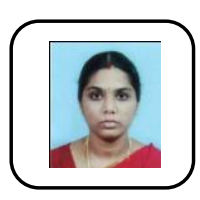

Dr. S.Shanthi, Assistant Professor,Department of Civil Engineering, Avinashilingam Institute for Home Science and Higher Education for Women , Completed B.E in Bharathiyar University, M.E in Anna University. Have published journal papers in UGC approved journals and also presented papers in International Conferences. Life time member of ICI. Have 14 years of teaching experience and 2 years of research experience
[8] Nor Syamimi Musa and Wan Azlina Ahmad , "Chemical Oxygen Demand Reduction in Industrial Wastewater using locally isolated Bacteria", Journal of Fundamental Sciences, Vol 6, No 2, Pp:8892, Nov 2010.

[9] Xinwei Yang, Jianqiao Gong, Wenxiao Liu, “Anaerobic Biological Treatment of high Concentration Wastewater", Chemical Engineering Transcations, Vol 71, Pp: 1423-1428, 2018

[10] OzgurKisi and Erdal Uncuoglu, "Comparison of Three Back Propagation Training algorithms for Two Case Studies”,, Indian Journal of Enginerring and Materials Sciences, $\quad$ Vol 12, Pp: 434442 , Oct 2005.

[11] S. Rama Subbanna, Dr. M. Suryakalavarthi , "Performance Analysis of ANN Training Algorithms to Detect the Magnetization Level in the Magnetic Core of a Welding Transformer", IOSR- JEEE, Vol 6, Issue 6, , Pp: 17-25, Jul- Aug 2013.

[12] R.Vijayabhanu and V. Radha , "Recognition and Elimination of Missing Values and Outliners From An Anaerobic Wastewater Treatment System using K-Means Cluster", , IEEE 3rd International Conference on Advanced Computer Theory and Engineering, Vol 4, pp 20-22, August 2010.

[13] Rabee Rustum and Adebaye J. Adeloye, "Replacing Outliers and Missing Values from Activated Sludge Data using Kohonen Self-Organizing Map", Journal Of Environmental Engineering (C) ASCE, Vol 133, Pp: 909-916, Sep 2007

[14] Tapas Kanungo, David M. Mount, Nathan S. Netanyahu, Christine D. Piatko, Ruth Silverman and Angela Y. Wu, "An Efficient K-Means Clustering Algorithm: Analysis and Implementation", IEEE Transactions on Pattern Analysis and Machine Intelligence, Vol 24, No. 7, Pp: 881- 892, July 2002.

[15] Jing-Ru Zhang, Jun Zhang, Tat-Ming Lok, Michael R. Lyu, “A Hybrid Particle Swarm Optimization-Back-Propagation Algorithm for Feed forward Neural Network Training", Applied Mathematics and Computations, Vol 185, Pp: 1026-1037, Feb 2007

[16] Zafer Comert, Adnan Fatih Kocamaz , 'A Study of Artificial Neural Network Training Algorithms for Classification of Cardiotocography Signals", Bitis Eren University of Science and Technology, Vol 7 (2), Pp: 93-103, Dec 2017

[17] R. Vijayabhanu and V. Radha, "Prediction Accuracy of BPN by Levenberg- Marquardt Algorithm for the Prediction of COD from an Anaerobic Reactor", $4^{\text {th }}$ International Conference on Signal and Image Processing, Springer, Vol 2, Pp: 571-581, December,2012 